\title{
1 Visualization of axonal protein allocation in Drosophila with whole-brain
}

\section{2 localization microscopy}

3 Authors: Li-An Chu ${ }^{1,2, \#}$, Chieh-Han $\mathrm{Lu}^{2,4, \#, \dagger}$, Shun-Min Yang ${ }^{2}$, Kuan-Lin Feng ${ }^{3}$, Yen-Ting

4 Liu ${ }^{4}$, Chun-Chao $\mathrm{Chen}^{1}$, Yun-Chi Tsai ${ }^{4}$, Peilin $\mathrm{Chen}^{4}$, Ting-Kuo Lee ${ }^{2}$, Yeu-Kuang Hwu ${ }^{2}$, Bi-

5 Chang Chen ${ }^{4 *}$, Ann-Shyn Chiang ${ }^{1,2,3,5,6,7^{*}}$

6 Affiliations:

7 1. Brain Research Center, National Tsing Hua University, Hsinchu 30013, Taiwan

8 2. Institute of Physics, Academia Sinica, Taipei 11529, Taiwan

9 3. Institute of Systems Neuroscience, National Tsing Hua University, Hsinchu 30013, 10 Taiwan

11 4. Research Center for Applied Sciences, Academia Sinica, Taipei 11529, Taiwan

12 5. Department of Biomedical Science and Environmental Biology, Kaohsiung Medical 13 University, Kaohsiung 80780, Taiwan

14 6. Institute of Molecular and Genomic Medicine, National Health Research Institutes, Zhunan, Miaoli 35053, Taiwan

7. Kavli Institute for Brain and Mind, University of California at San Diego, La Jolla, CA 92093-0526, USA

18 \#These authors contributed equally to the project.

$19 \dagger$ Present address: Department of Genetics and Complex Diseases, Harvard T H Chan School of Public Health, Boston, MA02115, United States

*Correspondence and requests for materials should be addressed to A.S.C. or B.C.C

22 Ann-Shyn Chiang, Brain Research Center, National Tsing Hua University, 
1 Bi-Chang Chen, Research Center for Applied Sciences, Academia Sinica,

2128 Sec. 2, Academia Rd., Nankang, Taipei 11529, Taiwan

3 Phone: 886-2-27873133; FAX: 886-2-27873122

4 


\section{$1 \quad$ Abstract}

2 Long-term memory (LTM) formation requires learning-induced protein synthesis in

3 specific neurons and synapses within a neural circuit. Precisely how neural activity

4 allocates new proteins to specific synaptic ensembles, however, remains unknown. We

5 developed a deep-tissue super-resolution imaging tool suitable for single-molecule

6 localization in intact adult Drosophila brain, and focused on the axonal protein allocation

7 in mushroom body (MB), a central neuronal structure involved in olfactory memory

8 formation. We found that insufficient training suppresses LTM formation by inducing the

9 synthesis of vesicular monoamine transporter (VMAT) proteins within a dorsal paired

10 medial (DPM) neuron, which innervates all axonal lobes of the MB. Surprisingly, using

11 our localization microscopy, we found that these learning-induced proteins are distributed

12 only in a subset of DPM axons in specific sectors along the MB lobes. This neural

13 architecture suggests that sector-specific modulation of neural activity from MB neurons

14 gates consolidation of early transient memory into LTM. 


\section{Introduction}

2 Memory formation requires learning-induced protein synthesis in specific neurons and 3 synapses within a neural circuit. In all species examined, there are two distinct phases of 4 memory formation: a transient neural activity associated with early memory and a protein5 synthesis-dependent change in synaptic connectivity associated with long-term memory $6(\mathrm{LTM})^{1,2}$. Early memory is labile; sustained neural activity during this phase nonetheless is 7 crucial for the induction of LTM and its underlying protein synthesis, which occurs only in a 8 few neurons sparsely distributed throughout the brain $^{3-5}$. Exactly how neural activity induces

9 protein synthesis in some but not all neurons in a circuit and then allocates new proteins to 10 specific synaptic ensembles during LTM formation, however, remains unknown.

Recent advances in super-resolution microscopy have allowed for localization of single molecules within individual cells ${ }^{6-8}$, but not within large tissues ${ }^{9}$. Our understanding of memory formation may benefit from these advances by enabling us to visualize the allocation of associative learning-induced proteins at the level of the synapse. In the present study, we integrated several optical technologies to develop a deep-tissue imaging tool suitable for single-molecule localization in an intact adult Drosophila brain. We show that insufficient training suppresses LTM formation by inducing the synthesis of vesicular monoamine transporter (VMAT) proteins in a single dorsal paired medial (DPM) neuron, which innervates all axonal lobes of the mushroom body (MB). Consistent with this observation, downregulation of VMAT or reduced serotonin synthesis in the DPM neuron enhances LTM

21 formation. Strikingly, we found that training-induced VMAT proteins are preferentially allocated to a specific subset of DPM neurites, which arborize within the $\alpha 2 / \beta^{\prime} 1 \mathrm{MB}$ sectors.

23 This neural architecture suggests that sector-specific serotonin modulation of neural activity 24 from MB neurons gates consolidation of early transient memory into LTM. Moreover, the 
1 present study demonstrates that our single-molecule imaging technique can be used to

2 visualize memory allocation at specific synaptic ensembles within an intact brain.

\section{Results}

\section{Deep-tissue localization microscopy (DTLM)}

5 The study of memory formation requires novel tools for visualizing the allocation of

6 learning-induced proteins into synaptic ensembles in an intact brain. Several research groups

7 have attempted to modify super-resolution microscopy techniques for use with larger imaging

8 volumes ${ }^{10-13}$. Among these techniques, point accumulation for imaging in nanoscale

9 topography has achieved sub-100 nm resolution in samples over $20-\mu \mathrm{m}$ thick by utilizing the

10 inherent optical sectioning of the lattice lightsheet to prevent premature photo-bleaching

11 (which limits localization and image quality) ${ }^{13,14}$. This method nonetheless fails to

12 compensate for tissue-induced aberrations, thereby restricting imaging depth and volume. To

13 visualize protein molecules within larger tissues, we integrated a Bessel beam lightsheet,

14 spontaneous blinking fluorophore, and optical tissue clearing to develop a deep-tissue

15 imaging tool suitable for single-molecule localization in an intact adult Drosophila brain (Fig.

16 1a and Methods). The lightsheet was generated by scanning a Bessel beam created by

17 filtering the laser illumination with an annular ring mask at the Fourier plane, which was

18 conjugated to the back aperture of the excitation objective (customized, N.A. $=0.5$, working

19 distance $=12.8 \mathrm{~mm}$; NARLabs, ITRC, Taiwan; Fig. 1b) ${ }^{15,16}$. The Bessel beam has been described as a self-reconstruction light beam that is particularly effective for penetrating into

21 a thick specimen ${ }^{15,16}$. The length of this lightsheet was extended from $50 \mu \mathrm{m}$ to over $200 \mu \mathrm{m}$ using an axicon lens (Supplementary Fig. 1a,b). Through constructive interference at the

23 imaging plane, the energy distribution of the lightsheet is spatially confined within a $0.5-\mu \mathrm{m}$

24 layer, thereby reducing background signal and preventing unwanted photo-bleaching 


\section{1 (Supplementary Fig. 1c,d).}

2 Traditional localization microscopy relies on two chemical mechanisms for the partial

3 activation of fluorophores: either an alternating two-wavelength exposure or a single-

4 wavelength high-intensity illumination ${ }^{7,8,17}$. Use of an additional activation laser, however, 5 prolongs image acquisition time and causes additional photo-bleaching due to short-

6 wavelength exposure. For high intensity illumination, the laser fluence deposited to the

7 sample rapidly consumes the photon budget and makes it unrealistic to achieve large scale

8 imaging. We addressed these problems by using a novel spontaneous blinking fluorophore,

9 HMSiR, which can be excited at a relatively low power density of $40 \mathrm{~W} / \mathrm{cm}^{2}$. Consequently

10 fluorophore blinking was extended to an area up to $75,000 \mu \mathrm{m}^{2}$ (Fig. 1b). When combined 11 with the use of HMSiR and tissue clearing (ScaleView-A2) ${ }^{18}$, our DTLM method can 12 reconstruct super-resolution images of an entire adult brain (Methods). Importantly, the 13 uncertainty of localized blinking events is similar through the entire $\mathrm{z}$ depth (Fig. 1c).

14 Typically, over $100 \mathrm{M}$ particles are contained in each of the four sub-volumes used to 15 reconstruct DTLM images of the whole brain (Supplementary Fig. 1e), and the total acquisition time is less than one day (Methods). To analyze gigantic datasets of raw images

17 and reconstruct the whole fly brain at super-resolution, we created a parallel computing 18 pipeline based on ThunderSTORM ${ }^{19}$ on a Lustre-backed Torque cluster (Supplementary

19 Movie 1, Supplementary Fig. 2).

20 The photoelectric sensors used for conventional fluorescence imaging (green fluorescent 21 protein, GFP) have a limited dynamic range. Thus, it can be difficult to capture structures with weaker GFP signals in a single image (Supplementary Fig. 5b, left). Because DTLM localizes individual molecules separately and reconstructs the image based on localization events, it is insensitive to intensity differences, thereby enabling the capture of greater detail in a single image. DTLM, for instance, captured a majority of parallel, densely bundled 
1 neural fibers connecting the brain and body (Supplementary Fig. 5b, middle). When

2 sufficient localization density was achieved, individual brain-ascending/descending fibers

3 could be digitally segmented (Supplementary Fig. 5b, right). Furthermore, DTLM enabled

4 three-dimensional visualization of individual synaptic proteins (Down syndrome cell

5 adhesion molecules) within a single spine-like protrusion in a dendrite of the giant fiber

6 neuron (Supplementary Fig. 5c).

\section{$7 \quad$ Visualizing fine neurites in the whole brain}

8 Targeted genetic manipulations using the promoter-driven $T H$-Gal4 line ${ }^{20}$ have revealed that

9 dopaminergic neurons (DANs) are involved in various brain functions in Drosophila,

10 including decision making ${ }^{21}$, arousal $^{22}$, and learning and memory ${ }^{23}$. To obtain a more

11 comprehensive understanding of these neural circuits underlying various behaviors, we used

12 single-molecule DTLM imaging to map the morphology and wiring patterns of all TH-Gal4

13 neurons in the adult brain (Fig. 2). Due to nominal photo-bleaching and optical clearing,

14 DTLM allowed us to image several overlapped sub-volumes under a high N.A. lens and

15 stitch them into a single big dataset of the entire brain at super-resolution. This large-volume

16 super-resolution map enabled simultaneous visualization of putative dopaminergic neurons

17 labelled with strong GFP signal in the central brain (Supplementary Fig. 6), as well as fine

18 neurites labelled with weak GFP signal in the optic lobe (Fig. 2a). Serial optical slices and

19 three-dimensional navigation demonstrated extensive yet separable neurites (Supplementary

20 Movie 3). These high-quality images also allowed for digital segmentation of most individual

21 neurons, with nominal axial overlap (Fig. 2b). Next, we applied DTLM to visualize the

22 allocation of learning-induced new proteins. 
1 Drosophila can learn to associate an odor with foot-shock punishment, and memory

2 formation thereafter exhibits several temporal phases, including protein synthesis-dependent

3 long-term memory ${ }^{24,25}$. The odor-shock association appears to occur in the MB, where the

4 conditioned odor stimulus is represented by the neural activity of a sparse subpopulation of

5 intrinsic Kenyon cells (KCs). The MB lobes can be subdivided into 15 consecutive sectors

6 based on their innervation by distinct types of DANs and MB output neurons (MBONs).

7 Evidence suggests that the unconditioned aversive stimulus is relayed via three types of

8 dopaminergic neurons innervating the $\alpha^{\prime} 1 \gamma^{2}, \beta 2 \beta^{\prime} 2$, and $\gamma 1$ sectors, respectively, thereby

9 modulating synaptic strength between $\mathrm{KCs}$ and $\mathrm{MBONs}$ and leading to conditioned

10 behaviours ${ }^{4,5,26-29}$. Multiple sessions of training with regular rest intervals (i.e., spaced

11 training) induce a transient increase in $\mathrm{KC}-\mathrm{MBON}$ responses that eventually transforms into

12 stable LTM - a process that involves local protein synthesis in three types of MBONs

13 innervating the $\alpha 3 / \beta^{\prime} 1 / \beta^{\prime} 2 / \gamma^{3}$ sectors, respectively ${ }^{4,5}$. It remains unclear how this sector-

14 specific modulation occurs and leads to LTM storage in specific neurons and synapses

15 downstream of the MB.

The dorsal paired medial (DPM) neuron, on the other hand, is a single giant neuron with extensive neurites that innervate all $\mathrm{MB}$ lobes, modulating KC-MBON activity by releasing serotonin to sustain neural activity associated with an intermediate phase of anesthesiaresistant memory $(\mathrm{ARM})^{27}$. In aged flies, reduced DPM-MBON connectivity appears to impair protein synthesis-dependent $\mathrm{LTM}^{28}$. Thus, ARM and LTM may be mutually exclusive $^{28}$. To address how DPM participates in memory formation, we first trained flies with DPM serotonin levels reduced by adult-stage specific RNAi-mediated downregulation of synthetic enzymes. Normally, flies form maximal 1-day memory after 10 sessions of spaced training (10x spaced) but minimal memory after only three sessions of spaced training 
1 now was sufficient to produce maximal 1-day memory (Fig. 3a). This enhanced LTM lasted

2 for at least 4 days and was not seen after 3x massed training (Fig. 3a), suggesting a bona-fide

3 protein-synthesis dependent LTM (and not ARM). With immunolabeling, we also observed a

4 significant increase in vesicular monoamine transporter (VMAT) proteins-which uptake

5 serotonin and other monoamines into presynaptic vesicles - at the DPM soma within 3 hours

6 after 3x spaced training (Fig. 3b). This increase in VMAT expression was abolished by acute

7 activation of temperature-sensitive $\mathrm{RICIN}^{\mathrm{cs}}$, a ribosomal toxin that inhibits protein

8 synthesis $^{3,30,31}$. RNAi-mediated downregulation of VMAT yielded a decrease in anti-VMAT

9 intensity in the DPM soma (Fig. 3c) and also enhanced 1-day memory after $3 \mathrm{x}$ spaced

10 training (Fig. 3d). Together, these results suggest that insufficient spaced training suppresses

11 LTM formation by inducing VMAT synthesis and increasing serotonergic signaling from

12 DPM neurons.

\section{Visualizing synaptic VMAT molecules in DPM neurites}

14 To further examine how insufficient training-induced VMAT proteins regulate serotonin 15 release from DPM neurons in synapses, we used single-molecule DTLM imaging to quantify 16 changes in VMAT distribution among all DPM neurites before and after 3x spaced training 17 (Fig. 4a). By cropping the MB boundary, we determined the total number of immunolabeled VMAT molecules in the MB. We classified these VMAT molecules into DPM+ and DPMgroups based on the 3D digital intersection between VMAT and DPM (Supplementary

Movie 4). Importantly, this classification can be achieved only by using DTLM

(Supplementary Fig. 7a) and cannot be achieved with state-of-the-art confocal microscopy

(Supplementary Fig. 7b). The precision of DPM+ VMAT localization was demonstrated by targeted $V M A T^{R N A i}$ expression, which reduced the total number of VMAT molecules in DPM+ neurites but not in the DPM- regions of MB lobes (Fig. 4b, Supplementary Fig. 7c). 
$13 x$ spaced training and found that their distributions are highly variable among different flies,

2 with a tendency to increase after training (Supplementary Fig. 7d). Unexpectedly, this

3 tendency was statistically insignificant (Fig. $4 \mathbf{b}$ ), regardless of the increase in the soma (Fig.

43 3b). Connectomic electron microscopy (EM) tracing of the $\alpha 1 / \alpha 2 / \alpha 3 \mathrm{MB}$ sectors indicates

5 that the DPM neuron synapses with DANs/KCs/MBONs to form intricate sector-specific

6 networks ${ }^{32,33}$. These DPM neurites exhibit branch-specific neural activity (memory traces)

7 after associative learning ${ }^{34}$, prompting us to investigate whether $3 \mathrm{x}$ spaced training induced

8 VMAT expression in some but not all sectors of DPM neurites.

$9 \quad$ MB sector-specific increases in DPM VMAT molecules induced by space training

10 We manually segmented DPM neurites into 15 MB sectors based on methods utilized in a 11 previous study ${ }^{35}$ (Supplementary Fig. 8 and Methods). Quantitative analysis revealed that

12 the density of VMAT molecules in DPM+ neurites increased after training in the $\alpha 2$ and $\beta^{\prime} 1$

13 sectors but not in the remaining MB sectors or in DPM- regions, when compared to that in 14 naïve flies (Fig. 4c, d). These increases appeared to be evenly distributed in the affected sectors. In naive flies that had not undergone training, the distribution of VMAT molecules was highly variable between DPM+ and DPM- neurites and among different sectors (Fig. 4c).

\section{Discussion}

18 Spatiotemporal allocation of associative learning-induced VMAT molecules suggests that 19 DPM neurons regulate memory formation via serotonergic modulation of DAN/KC/MBON 20 circuits in specific MB sectors. Translational regulation may account for this sector-specific 21 allocation, as increases in VMAT are (i) regulated by Fragile $\mathrm{X}$ mental retardation protein, 22 which itself is involved in olfactory LTM formation ${ }^{36,37}$ and (ii) blocked by inhibition of 23 protein synthesis (Fig. 3d). Transcriptional regulation may also be implicated, as increases in

24 VMAT expression after training occur in both the soma and neurites of DPM neurons (Figs. 
13 and 4). Previous studies have suggested that training induces local protein synthesis in

$2 \alpha 3 / \beta^{\prime} 1 / \beta^{\prime} 2 / \gamma 3$ MBONs during LTM consolidation ${ }^{4,5}$. The findings of the present study

3 suggest that modulation of serotonergic signaling (via VMAT) from DPM neurons to sector-

4 specific MB neurons also contributes to LTM consolidation during the first few hours after

5 training (Fig. 3d) but not during LTM retrieval ${ }^{38}$. Further studies are required to determine

6 whether $\alpha 2 / \beta^{\prime} 1-$ VMATs in the DPM directly and specifically modulate the function of

$7 \alpha 2 / \beta^{\prime} 1$ MBONs. Although $\alpha 2$ MBONs exhibit highly variable functional responses among

$8 \quad$ individual subjects ${ }^{39}$, their outputs appear necessary during LTM retrieval ${ }^{39}$. In contrast, $\beta^{\prime} 1$

9 MBONs appear to be involved in the forgetting process ${ }^{40}$.

10 Neural plasticity involves local protein synthesis in both presynaptic axon terminals and 11 postsynaptic dendrite spines $^{41}$. In Drosophila olfactory memory formation, postsynaptic

12 regulation of protein synthesis has been demonstrated ${ }^{4,5}$, and here we present data suggesting

13 presynaptic regulation of protein synthesis during memory formation, as well. Using deep-

14 tissue localization microscopy, we further extend the visualization of protein allocations in a

15 few target neurons to a systems level of analysis. Consistent with branch-specific functional 16 responses during associative learning ${ }^{34}$, our finding of sector-specific allocation of

17 presynaptic VMAT proteins in DPM neurons adds another layer of coding complexity to the

$18 \mathrm{DANs} / \mathrm{KCs} / \mathrm{MBONs}$ circuit within the MB. Spatiotemporal VMAT-mediated serotonin

19 release from DPM axons then directly modulates the neural activity of a subset of KCs within

20 specific MB sectors. Such serotonergic signaling helps to translate transient neural activity in 21 the MB circuit during early memory into LTM that includes postsynaptic regulation of 22 protein synthesis in specific MBONs.

23 One limitation of our current imaging method is that the precision of axial localization is 24 restricted by the thickness of the Bessel lightsheet $(\sim 500 \mathrm{~nm})$, which is much less than the 25 precision at the lateral plane $(\sim 30 \mathrm{~nm})$. Thus, separating entangled neurites is trivial at $\mathrm{X}-\mathrm{Y}$ 
1 plane but not always reliable along the $\mathrm{Z}$ axis. In this study, we corrected for this Z-plane

2 limitation by classifying VMAT immune-positive signals into DPM+ versus DPM- groups.

3 This approach was reasonably reliable because all imaged neurites in the MB derived from a

4 single DPM neuron. In future studies, the Z-axis resolution may be improved further (i) by 5 optical clearing using a medium with a higher refractive index ${ }^{42}$ matched with a higher-

6 aperture objective, (ii) by using a cylindrical lens to refine the $\mathrm{Z}$ position ${ }^{43}$, and/or (iii) by

7 combining DTLM with expansion microscopy ${ }^{44}$. With imaging near isotropic super-

8 resolution, visualization of the connectome representing LTM-dependent neuroplasticity

9 among all synaptic connections in an intact fly brain soon will be achievable ${ }^{3-5}$.

\section{References}

121 Kandel, E. R., Dudai, Y. \& Mayford, M. R. The molecular and systems biology of 13 memory. Cell 157, 163-186 (2014).

142 Tonegawa, S., Pignatelli, M., Roy, D. S. \& Ryan, T. J. Memory engram storage and 15 retrieval. Curr Opin Neurobiol 35, 101-109 (2015).

163 Chen, C. C. et al. Visualizing long-term memory formation in two neurons of the 17 Drosophila brain. Science 335, 678-685 (2012).

184 Pai, T. P. et al. Drosophila ORB protein in two mushroom body output neurons is 19 necessary for long-term memory formation. Proc Natl Acad Sci U S A 110, 7898-7903 $20 \quad$ (2013).

$215 \mathrm{Wu}$, J. K. et al. Long-term memory requires sequential protein synthesis in three subsets 22 of mushroom body output neurons in Drosophila. Sci Rep 7, 7112, doi:10.1038/s41598017-07600-2 (2017). 
16 Hell, S. W. \& Wichmann, J. Breaking the diffraction resolution limit by stimulated

2 emission: stimulated-emission-depletion fluorescence microscopy. Opt Lett 19, 780-782 (1994).

47 Rust, M. J., Bates, M. \& Zhuang, X. Sub-diffraction-limit imaging by stochastic optical 5 reconstruction microscopy (STORM). Nat Methods 3, 793-795 (2006).

68 Betzig, E. et al. Imaging intracellular fluorescent proteins at nanometer resolution. $7 \quad$ Science 313, 1642-1645 (2006).

89 Spuhler, I. A., Conley, G. M., Scheffold, F. \& Sprecher, S. G. Super resolution imaging 9 of genetically labeled synapses in Drosophila brain tissue. Front Cell Neurosci 142, 1-10 $10 \quad$ (2016).

1110 Vaziri, A., Tang, J., Shroff, H. \& Shank, C. V. Multilayer three-dimensional super 12 resolution imaging of thick biological samples. Proc Natl Acad Sci U S A 105, 20221$1320226(2008)$.

1411 Abrahamsson, S. et al. Fast multicolor 3D imaging using aberration-corrected multifocus microscopy. Nat Methods 10, 60-63 (2013).

12 Cella Zanacchi, F. et al. Live-cell 3D super-resolution imaging in thick biological samples. Nat Methods 8, 1047-1049 (2011).

1813 Legant, W. R. et al. High-density three-dimensional localization microscopy across large 19 volumes. Nat Methods 13, 359-365 (2016).

14 Schueder, F. et al. Multiplexed 3D super-resolution imaging of whole cells using spinning disk confocal microscopy and DNA-PAINT. Nat Commun 8, 2090 (2017). beams enables contrast-enhanced imaging in thick media. Nat Commun 3, 632 (2012). 
116 Gao, L., Shao, L., Chen, B. C. \& Betzig, E. 3D live fluorescence imaging of cellular

2 dynamics using Bessel beam plane illumination microscopy. Nat Protoc 9, 1083-1101

3 (2014).

417 Heilemann, M. et al. Subdiffraction-resolution fluorescence imaging with conventional $5 \quad$ fluorescent probes. Angew Chem Int Ed Engl 47, 6172-6176 (2008).

618 Hama, H. et al. Scale: a chemical approach for fluorescence imaging and reconstruction 7 of transparent mouse brain. Nat Neurosci 14, 1481-1488, (2011)

819 Ovesny, M., Krizek, P., Borkovec, J., Svindrych, Z. \& Hagen, G. M. ThunderSTORM: a 9 comprehensive ImageJ plug-in for PALM and STORM data analysis and super10 resolution imaging. Bioinformatics 30, 2389-2390 (2014).

20 Friggi-Grelin, F. et al. Targeted gene expression in Drosophila dopaminergic cells using regulatory sequences from tyrosine hydroxylase. J Neurobiol 54, 618-627 (2003).

21 Zhang, K., Guo, J. Z., Peng, Y., Xi, W. \& Guo, A. Dopamine-mushroom body circuit regulates saliency-based decision-making in Drosophila. Science 316, 1901-1904 (2007).

22 Andretic, R., van Swinderen, B. \& Greenspan, R. J. Dopaminergic modulation of arousal in Drosophila. Curr Biol 15, 1165-1175 (2005).

23 Waddell, S. Reinforcement signalling in Drosophila; dopamine does it all after all. Curr Opin Neurobiol 23, 324-329 (2013).

24 Dubnau, J. \& Chiang, A. S. Systems memory consolidation in Drosophila. Curr Opin Neurobiol 23, 84-91 (2013).

25 Cognigni, P., Felsenberg, J. \& Waddell, S. Do the right thing: neural network mechanisms of memory formation, expression and update in Drosophila. Curr Opin Neurobiol 49, 51-58 (2018).

26 Aso, Y. et al. Three dopamine pathways induce aversive odor memories with different stability. PLoS Genet 8, e1002768 (2012). 
127 Lee, P. T. et al. Serotonin-mushroom body circuit modulating the formation of anesthesia-resistant memory in Drosophila. Proc Natl Acad Sci U S A 108, 13794-13799 (2011).

28 Isabel, G., Pascual, A. \& Preat, T. Exclusive consolidated memory phases in Drosophila. Science 304, 1024-1027 (2004).

29 Tonoki, A. \& Davis, R. L. Aging impairs protein-synthesis-dependent long-term memory in Drosophila. J Neurosci 35, 1173-1180 (2015).

30 Endo, Y., Mitsui, K., Motizuki, M. \& Tsurugi, K. The mechanism of action of ricin and related toxic lectins on eukaryotic ribosomes. The site and the characteristics of the modification in $28 \mathrm{~S}$ ribosomal RNA caused by the toxins. $J$ Biol Chem 262, 5908-5912 (1987).

31 Endo, Y. \& Tsurugi, K. RNA N-glycosidase activity of ricin A-chain. Mechanism of action of the toxic lectin ricin on eukaryotic ribosomes. $J$ Biol Chem 262, 8128-8130 (1987).

32 Aso, Y. \& Rubin, G. M. Dopaminergic neurons write and update memories with celltype-specific rules. Elife 5 (2016).

1733 Takemura, S. Y. et al. A connectome of a learning and memory center in the adult Drosophila brain. Elife 6 (2017).

34 Yu, D., Keene, A. C., Srivatsan, A., Waddell, S. \& Davis, R. L. Drosophila DPM neurons form a delayed and branch-specific memory trace after olfactory classical conditioning. Cell 123, 945-957 (2005).

35 Aso, Y. et al. The neuronal architecture of the mushroom body provides a logic for associative learning. Elife 3 (2014). 
136 Tauber, J. M., Vanlandingham, P. A. \& Zhang, B. Elevated levels of the vesicular monoamine transporter and a novel repetitive behavior in the Drosophila model of fragile X syndrome. PLoS One 6, e27100 (2011).

37 Bolduc, F. V., Bell, K., Cox, H., Broadie, K. S. \& Tully, T. Excess protein synthesis in Drosophila fragile X mutants impairs long-term memory. Nat Neurosci 11, 1143-1145 (2008).

38 Keene, A. C., Krashes, M. J., Leung, B., Bernard, J. A. \& Waddell, S. Drosophila dorsal paired medial neurons provide a general mechanism for memory consolidation. Curr Biol 16, 1524-1530 (2006).

39 Hige, T., Aso, Y., Rubin, G. M. \& Turner, G. C. Plasticity-driven individualization of 11 olfactory coding in mushroom body output neurons. Nature 526, 258-262 (2015).

40 Shuai, Y. et al. Dissecting neural pathways for forgetting in Drosophila olfactory aversive memory. Proc Natl Acad Sci U S A 112, E6663-6672 (2015).

41 Younts, T. J. et al. Presynaptic protein synthesis is required for long-term plasticity of GABA release. Neuron 92, 479-492 (2016).

42 Long, X., Colonell, J., Wong, A. M., Singer, R. H. \& Lionnet, T. Quantitative mRNA imaging throughout the entire Drosophila brain. Nat Methods 14, 703-706 (2017).

43 Huang, B., Wang, W., Bates, M. \& Zhuang, X. Three-dimensional super-resolution imaging by stochastic optical reconstruction microscopy. Science 319, 810-813 (2008). 
1 (chenb10@gate.sinica.edu.tw) or A.S.C. (aschinag@life.nthu.edu.tw).

\section{Acknowledgments} Academia Sinica for B.C.C. (Career Development Award). and managed the project. welcome to comment on the online version of the paper.

We are grateful to Tim Tully for critical comments and editing on the manuscript. The control software of the microscope is licensed by Howard Hughes Medical Institute, Janelia Farm Research Campus. We thank David Krantz for sharing the rabbit-anti VMAT antibody. We thank Vienna Drosophila Resource Center, Drosophila Genetic Resource Consortium and Bloomington Stock Center for fly stocks. This work was supported by grants from the Ministry of Science and Technology and Ministry of Education of Taiwan for L.A.C (MOST 106-2321-B-007-008-MY3), A.S.C. (Higher-Education Deep Cultivation Program) and B.C.C. (MOST 107-3017-F-007-004, MOST 103-2113-M-001-003-MY2), and from the

Author contributions: L.A.C. and C.H.L. planned and performed the imaging experiments and image processing. L.A.C. performed immunostaining experiments. C.H.L, Y.T.L, and B.C.C. designed and constructed the DTLM. S.M.Y. produced the HMSiR conjugates. K.L.F. and C.C.C. planned and performed the behavioral experiments. Y.T.L. designed the automated blinking image processing protocol and software. L.A.C. performed image analysis. L.A.C., C.H.L., B.C.C. and A.S.C. wrote the manuscript. B.C.C and A.S.C. planned

Competing interests: The authors declare no competing financial interests. Readers are

Data and materials availability: Requests for materials should be addressed to B.C.C. 


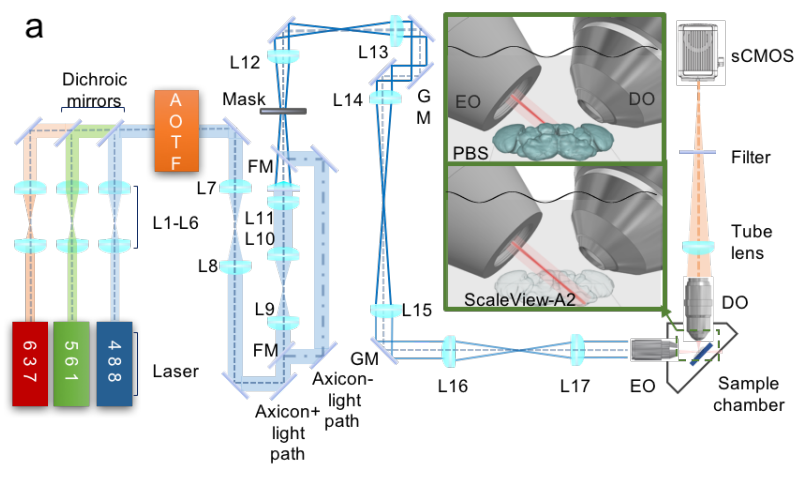

2

3 Fig. 1. Bessel beam lightsheet for deep-tissue localization microscopy (DTLM). (a) The

4 schematic configuration of the DTLM system. Inset: the geometry of relative positions ScaleView-A2. Scale bar $=1 \mu \mathrm{m}$.

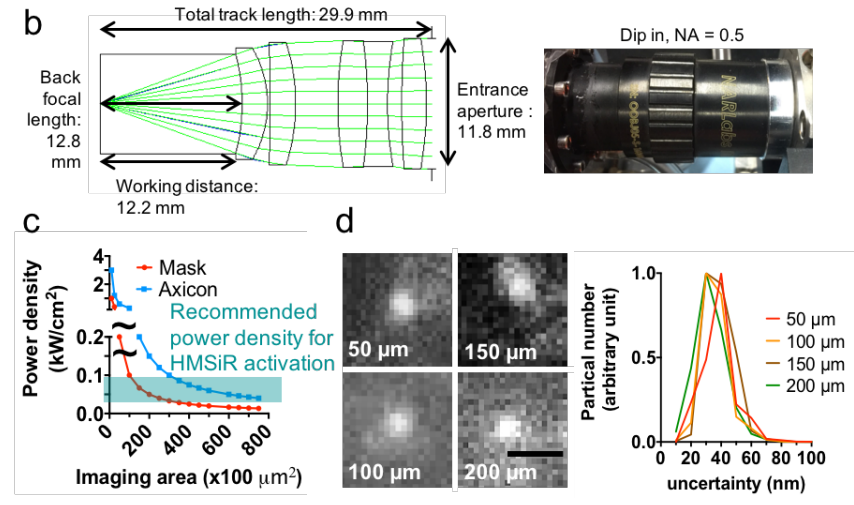

between two objective lenses and the specimen. AOTF: acoustic-optical tuneable filter; EO,

excitation objective; DO, detection objective; GM: galvo mirror; L: lens. (b) A customized

dip-in objective lens matching refractive index of ScaleView-A2 at a long working distance of $12.2 \mathrm{~mm}$. Left, Photograph of the lens. Right, Schematic design and simulated ray tracing.

(c) The lightsheet generated using an axicon lens carries higher power density than that generated using the mask only. At the power density $\left(40 \mathrm{w} / \mathrm{cm}^{2}\right)$ for HMSiR excitation, the maximum lightsheet areas are $25,000 \mu \mathrm{m}^{2}$ and $75,000 \mu \mathrm{m}^{2}$, generated using a mask only and an axicon lens, respectively. (d) Representative HMSiR blinking events (left) and overall lateral uncertainty distribution (right) at four different depths in the fly brain cleared with 


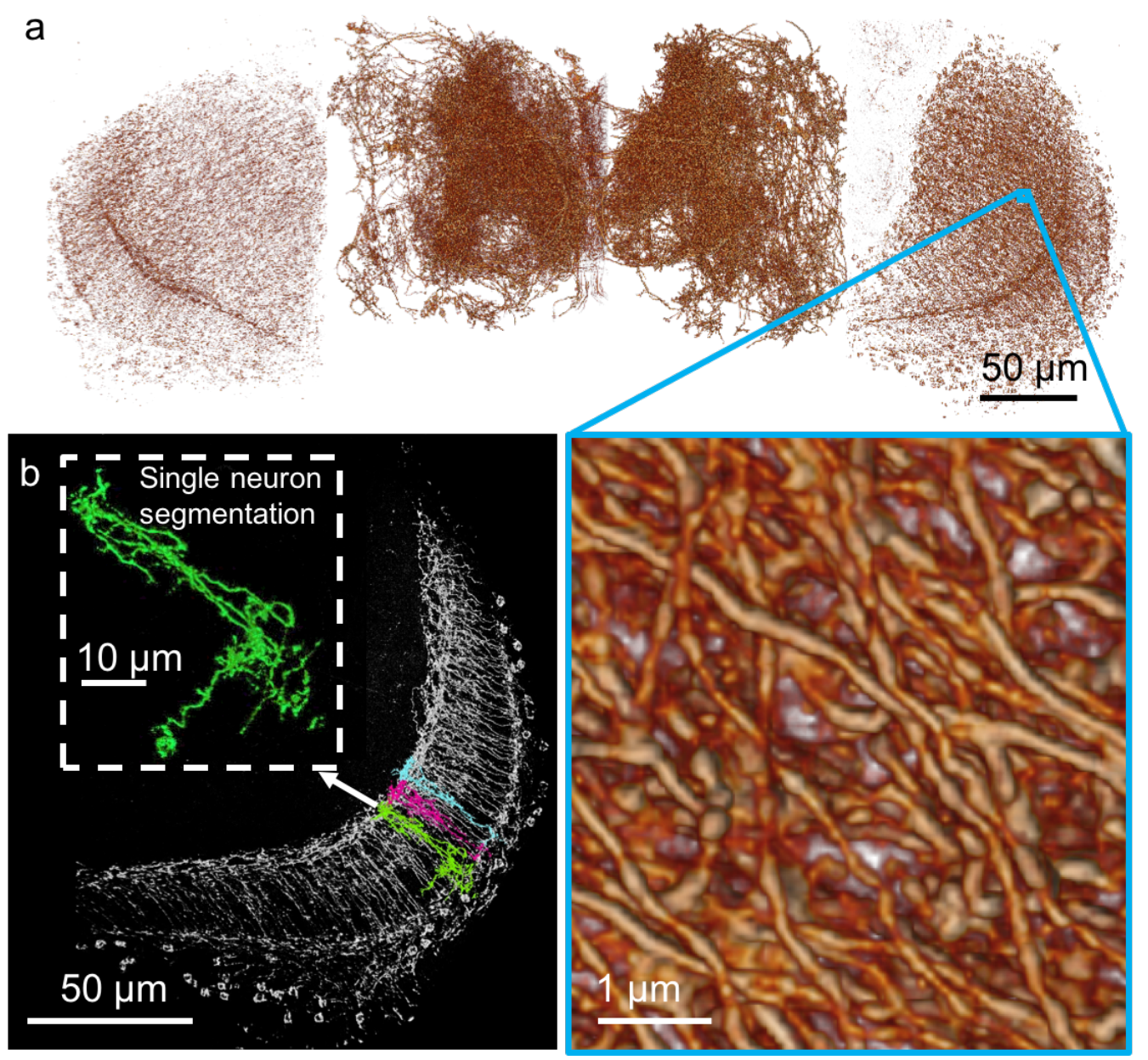

2 Fig. 2. Single-molecule deep-tissue localization microscopy (DTLM) imaging of

3 dopaminergic neurons (DANs) in the whole Drosophila brain. (a) Volume rendering of

4 whole-brain DANs. Zoom-in images show distinguishable interweaved neurites. (b) Digital

5 segmentation of local neurons in the medulla. Inset: enlarged green neuron. The

6 experimental flies carried TH-Gal4; UAS-GCaMP6f transgenes. 

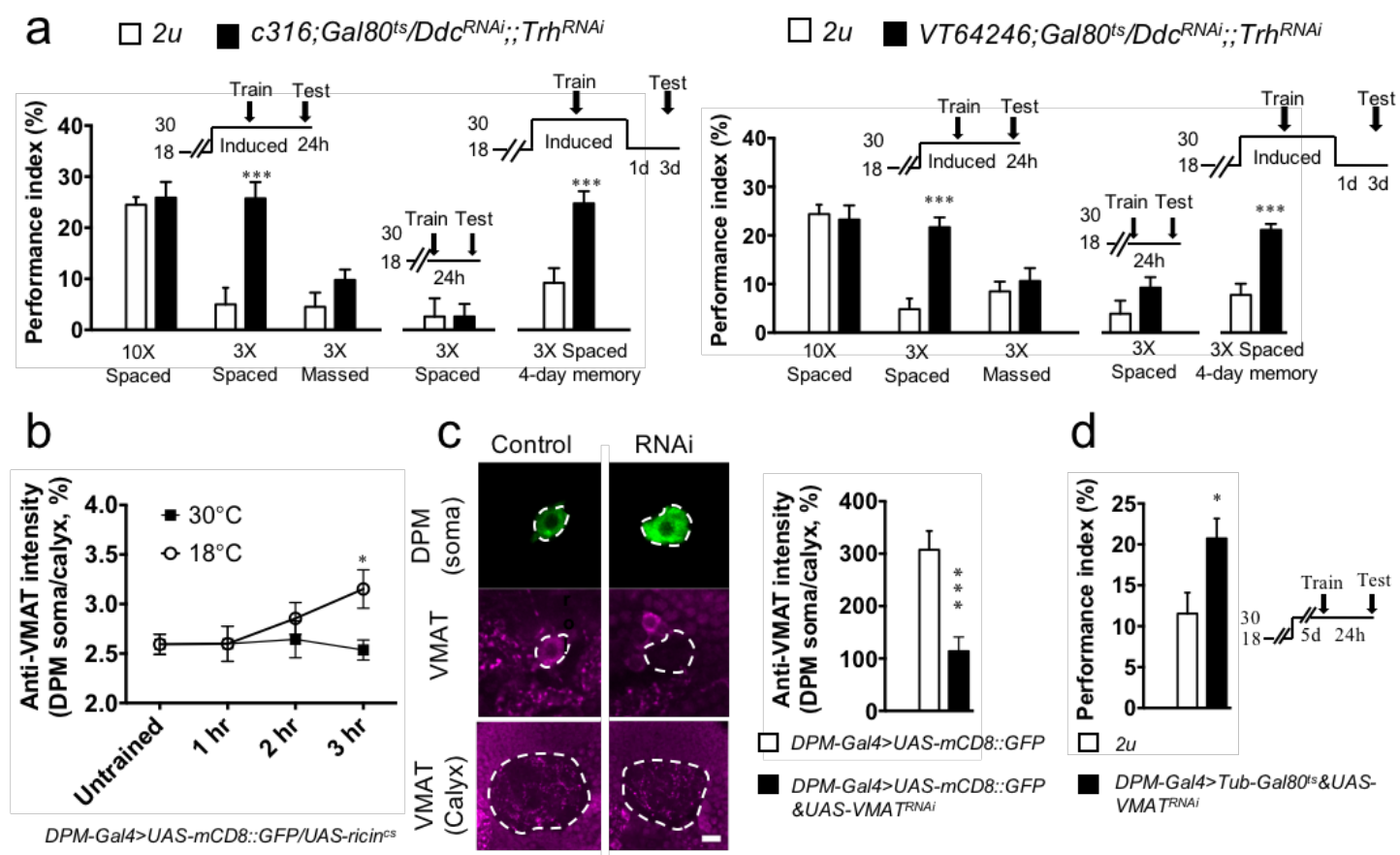

2 Fig. 3. Serotonin released from dorsal paired medial (DPM) neurons suppresses longterm memory (LTM) formation. (a) Effects of adult-stage specific down-regulation of

4 serotonin synthesis enzymes $\left(D d c^{R N A i}\right.$ and $\left.T r h^{R N A i}\right)$ with two independent DPM-Gal4 drivers 5 (c316 and VT64246) on 1-day and 4-day memory retention after various training protocols.

6 (b) Changes in VMAT signals in the DPM soma within 3 hours after training. Protein

7 synthesis was blocked by the active ribosomal toxin $\mathrm{RICIN}^{\mathrm{cs}}$ at $30^{\circ} \mathrm{C}$ but unaffected by the

8 inactive $\mathrm{RICIN}^{\mathrm{cs}}$ at $18^{\circ} \mathrm{C}$. (c) Effectiveness and specificity. Left, $V M A T^{R N A i}$ (vesicular

9 monoamine transporter) effectively downregulates anti-VMAT immuno-positive signals

10 (magenta) in the DPM cell body (green) but not in the mushroom body (MB) calyx, which is

11 not innervated by the DPM neuron. Right, Quantitative measurements. (d) Adult-stage

12 specific RNAi-mediated downregulation of VMAT in the DPM neuron enhanced 1-day

13 memory (DPM-Gal4: VT64246-Gal4) (see Methods for training protocols). Each value

14 represents the mean \pm S.E.M. $(\mathrm{n}=8$ in $\mathbf{A}, \mathbf{B}, \mathbf{D}, \mathrm{n}=10$ in $\mathbf{C}){ }^{*}, \mathrm{P}<0.05 ; * * *, \mathrm{P}<0.001$. 

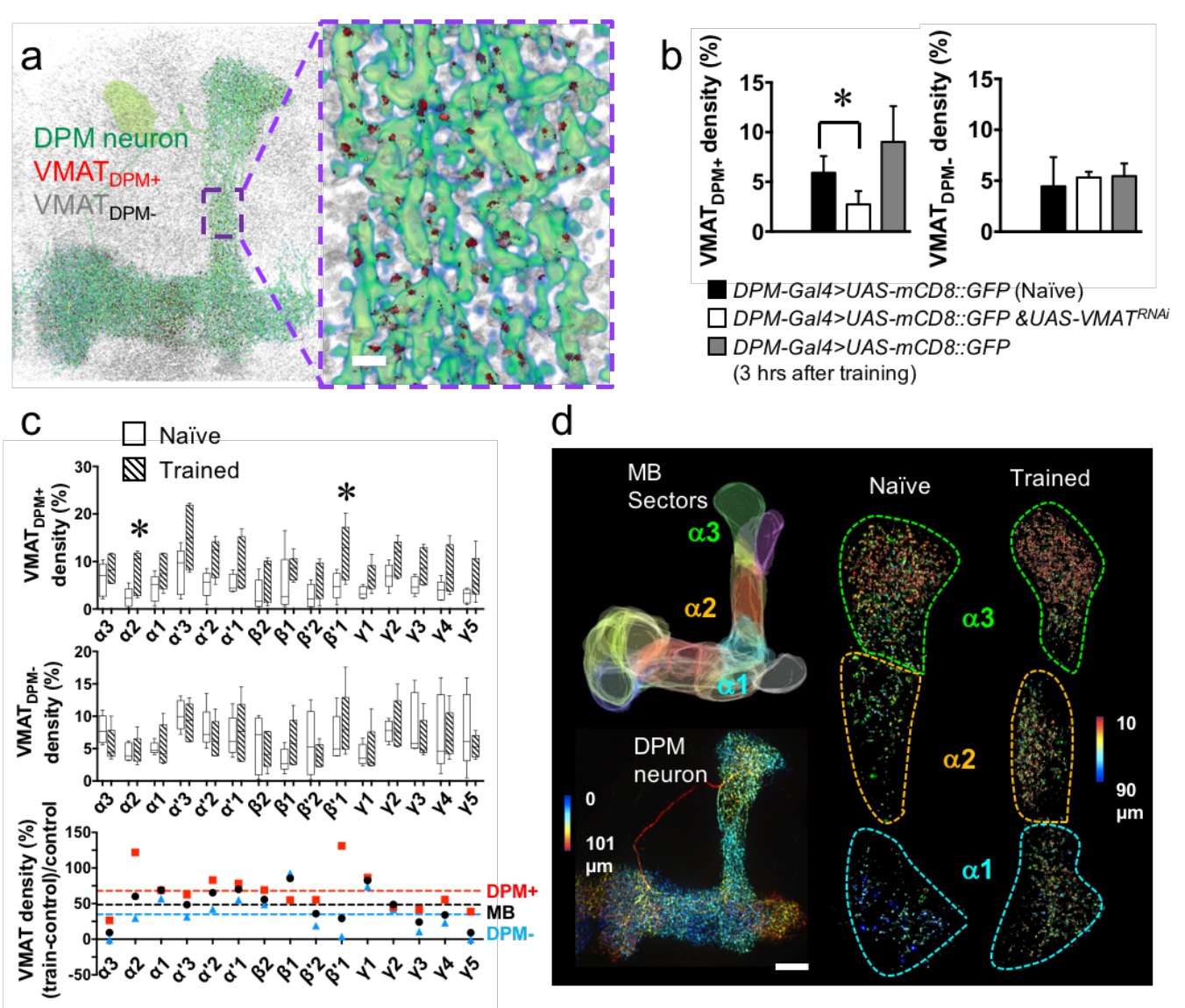

2 Fig. 4. Training induces sector-specific increases in VMAT expression in axons of DPM

3 neurons. (a) Visualization of VMAT molecules. Left, VMAT proteins (gray) distributed

4 within (red) or outside of (gray) the DPM neurons (green). Right, an enlarged view. (b)

$5 V M A T^{\text {RNAi }}$-mediated changes in VMAT density in the DPM+ neurites (left) and DPM- regions

6 (right) within the MB 3 hours after 3x spaced training. (c) Comparison of VMAT distribution

7 in DPM axons between naïve and trained flies. VMAT density increases $>100 \%$ in the $\alpha 2$

8 and $\beta^{\prime} 1$ sectors of DPM+ neurites (red) but not DPM- regions (blue) after 3x spaced training.

9 The dashed line indicates the average change among different sectors [Each value represents

10 the mean \pm S.E.M. $(\mathrm{n}=6$ in $\mathbf{B} ; \mathrm{n}=5$ in $\mathbf{C}) ; *=\mathrm{P}<0.05$; see Supplementary Fig. 8 for

11 calculations]. (d) Upper left, a schematic representation of MB sectors. Lower left, a single

12 DPM neuron innervates all MB lobes. Right, a representative volume image shows the 
1 training-induced increase in the number of VMAT proteins in the DPM neurites innervating

2 the $\alpha 2$ sector. Color code indicates depth.

3 


\section{Methods}

\section{Fly stocks}

3 Fly stocks were raised on cornmeal food at a temperature of $25^{\circ} \mathrm{C}$ and relative humidity of

$470 \%$ under a 12-h light/dark cycle. The following fly lines were used in the current study:

5 Fruitless-Gal4 (66696, Bloomington Drosophila Stock Center) was used to label neck

6 neurons, MZ19-Gal4 (34497, Bloomington Drosophila Stock Center) was used to label

7 olfactory projection neurons, 12862-Gal4 (111501, DGRC) was used to label giant fiber

8 neurons, TH-Gal4 (8848, Bloomington Drosophila Stock Center) was used to label

9 dopaminergic neurons, c316-Gal4 (30830, Bloomington Drosophila Stock Center) and

10 VT64246-Gal4 (v204311, VDRC) were used to label DPM neurons, UAS-mCD8::GFP (5137

11 and 5310, Bloomington Drosophila Stock Center)and UAS-GCaMP6f (42747, Bloomington

12 Drosophila Stock Center) were used as reporters of Gal4 expression, UAS-Dscam[1.7]::GFP

13 (From T. Lee, Howard Hughes Medical Institute, Ashburn, VA) was used to label Dscam in

14 Gal4-labelled neurons, tub-Gal80 $0^{\text {ts }}$ (From L. Luo, Stanford University, Stanford, CA) was

15 used to block Gal4 expression at $18^{\circ} \mathrm{C}, U A S-D d c^{R N A i}(3329, \mathrm{VDRC})$ and $U A S-T r h^{R N A i}(35240$,

16 VDRC) were used to downregulate serotonin expression in DPM neurons, and UAS-VMAT

$17 R N A i$ (v104072 and v4856, VDRC) was used to downregulate VMAT protein expression in

18 DPM neurons.

\section{Immunohistochemistry}

20 Fly brains were dissected in PBS ( $\mathrm{pH}$ 7.2) and immediately transferred to a microwave-safe

21 24-well plate containing 4\% paraformaldehyde in PBS. The plate was placed on a shaker for

2225 min. Fixed tissues were then permeabilized and blocked in PBS containing 2\% Triton X-

23100 and 10\% normal goat serum (NGS; Vector Laboratories, Burlingame, CA) at $4^{\circ} \mathrm{C}$ 
1 and $0.25 \%$ NGS using the following primary antibodies: mouse anti-Discs large antibodies

2 (antibody 4F3; 1:20 dilution; Developmental Studies Hybridoma Bank, Iowa City, IA), rabbit

3 anti-GFP antibodies (1:250 dilution; Thermo Fisher Scientific Inc, A11122), rabbit antiVMAT (1:250 dilution), and secondary antibodies including either biotinylated goat antirabbit immunoglobulin G (IgG) (1:250 dilutions; Thermo Fisher Scientific Inc). Biotinconjugated IgG was detected using Alexa Fluor streptavidin 635 (1:500 dilution; Thermo

7 Fisher Scientific Inc) or HMSiR streptavidin (1:1000 dilution). Each step was carried out over the course of 2 days, with extensive washes between steps at room temperature $\left(25^{\circ} \mathrm{C}\right)$.

9 Samples were then transferred to ScaleView-A2 for 2 days before imaging.

\section{$10 \quad$ HMSiR conjugates}

11 For goat anti-rabbit labelling of HMSiR, we incubated $250 \mu \mathrm{g}$ of goat anti-rabbit F(ab')2 12 fragment (Jackson Immunoresearch, West Grove, PA) in $0.1 \mathrm{M}$ sodium borate buffer at $\mathrm{pH}$ 138.5 with $0.8 \mu \mathrm{l}$ of $10 \mathrm{mM}$ HMSiR-NHS (GORYO Chemical, Bunkyo-ku, Tokyo) in DMSO at $37^{\circ} \mathrm{C}$ for $30 \mathrm{~min}$, after which the sample was incubated at $4^{\circ} \mathrm{C}$ overnight. The conjugated antibody was then passed through a Desalt Z-25 column (emp Biotech GmbH, Berlin) to remove unconjugated HMSiR-NHS, and the medium was replaced with PBS (pH 7.2). To label HMSiR with streptavidin, we incubated $125 \mu \mathrm{g}$ of streptavidin (Sigma-Aldrich, St. Louis, MO) in $250 \mu \mathrm{lBS}$ at $\mathrm{pH} 7.2$ with $0.8 \mu \mathrm{l}$ of $10 \mathrm{mM}$ HMSiR-NHS in DMSO, after which we utilized the same protocol as that used for antibody labelling. Concentrations were then measured at an absorbance of $280 \mathrm{~nm}$, after which the HMSiR conjugates were stored at $21 \quad 4^{\circ} \mathrm{C}$ until use.

\section{Microscope optics and image acquisition}

23 The lasers were combined with long-pass dichroic filters and aligned collinearly before entering an acousto-optical tunable filter (AOTF), which is used to control laser exposure 
1 time and wavelength. The laser beam passing through the AOTF was expanded to $4 \mathrm{~mm}$ full

2 width at half maximum (FWHM) to distribute the energy evenly onto the annular ring pattern

3 at the quartz mask for creating the illumination pattern conjugated to the back focal plane of

4 the objective lens. To conjugate the illumination pattern to the scanning mirrors, a pair of

5 lenses aligned in 4F geometry was inserted between the mask and scanning mirrors (L12,

6 L13, Fig. 1a). Another 4F lens pair was inserted between the galvo mirrors to relay the

7 pattern (L14, L15, Fig. 1a). After reaching the scanning mirror (Cambridge Technology,

$86215 \mathrm{H})$, the pattern was magnified and conjugated to the back aperture of the excitation

9 objective through another pair of lenses (L16, L17, Fig. 1a). A dip-in objective was placed

10 perpendicular to the illumination plane to collect the fluorescence signal. Using a piezo

11 scanner (Physik Instrumente, P-725.4 PIFOC), the detection objective was moved in

12 synchrony with the position of the lightsheet, which was controlled by the scanning mirror.

13 Between the individual volumetric scans, an additional settle time on the order of

14 milliseconds was included to stabilize the piezo scanner.

The length of the self-reconstructing axial extent of the Gaussian-Bessel lightsheet can be controlled by the geometry of the illumination pattern ${ }^{16}$. A ring-shaped illumination will

17 transform into a concentric irradiance profile distributed along the radial direction around the

18 optical axis at the focal plane of the excitation objective lens. The illumination profile and the

19 total power carried by the Bessel beam are controlled by the diameter and thickness of the 20 annular ring. In the present study, the geometry of the illumination pattern was chosen based 21 on a balance between the area of coverage and the power density of the lightsheet. To achieve 22 stochastic blinking with reasonable signal to noise ratio (SNR), it is necessary to fill the 23 observation plane with a power density above $40 \mathrm{~W} / \mathrm{cm}^{2}$. To localize subareas within the fruit 24 fly brain, we used a lightsheet with an axial FWHM of $50 \mu \mathrm{m}$ (outer N.A. $=0.26$, inner N.A. $25=0.185)$. 
1 As the size of the observation area increases, however, the power density of the lightsheet

2 created by simply filtering the laser profile using an annular ring mask is insufficient for

3 providing high precision localization because of the decreasing SNR, as the annular ring

4 intrinsically filters out the most intense component from the Gaussian irradiance profile (Fig.

5 1d2, e1). In this optical configuration, the field-of-view (FOV) cannot be extended further.

6 We therefore used an axicon lens (Thorlabs) to concentrate the laser energy into a ring

7 pattern, following which the unwanted components were filtered using an annular ring mask

8 (outer N.A. $=0.187$, inner N.A. $=0.174$ ). The length of the lightsheet generated by the axicon

9 lens can extend to over $200 \mu \mathrm{m}$ to cover the entire cross section of the fruit fly brain while maintaining a sufficient power density to excite HMSiR (Supplementary Fig. S1d3, e2).

Choice of the objective lens pair depends on the type of immersion medium. In PBS, a customized excitation objective (Special Optics, 0.65 NA, $3.74 \mathrm{~mm}$ WD) was used for image acquisition, while a water immersion detection objective lens (Nikon, CFI Apo LWD 25XW, 1.1 NA, 2 mm WD) was used for signal collection. For image acquisition in ScaleView-A2, the excitation objective lens was replaced by a customized objective lens (N.A. $=0.5$, working distance $=12.8 \mathrm{~mm}$; NARLabs, ITRC, Taiwan, Fig. 1b) designed to optimize performance in media with a higher refractive index $(n=1.38)$. An immersion detection objective (Olympus, XLPLN25XSVMP2, 25X, 1.0 NA) was used for single-molecule detection in the high-refractive index medium. In the present study, the lightsheet system was carefully calibrated to facilitate single-molecule detection with respect to each of the experimental conditions. As shown in Extended Data Fig. 1, the point spread function (PSF) values from different system configurations were compared to quantify the image quality of the lightsheet microscope system.

Single molecule fluorescence was detected using a sCMOS camera (Hamamatsu, Orca Flash 4.0 v2 sCOMS) equipped with a tube lens $(f=500 \mathrm{~mm})$. The exposure time of each 
1 frame is typically $100 \mathrm{~ms}$, while the period of an individual volume stack is approximately 40

$2 \mathrm{~s}$ (400 layers in one stack).

3 Samples were loaded onto 5-mm round glass coverslips (Warner Instruments) using 100

$4 \mathrm{nl}$ of Cell-Tak ${ }^{\mathrm{TM}}$ (Corning $\left.{ }^{\circledR}\right)$. During image acquisition, samples and both objectives were

5 immersed in a chamber to maintain optical clarity. All experiments were conducted at room

6 temperature.

7 The use of a Bessel beam lightsheet allows for single-molecule localization even when 8 tissues are maintained in PBS, although the uncertainty of molecule localization increases

9 along with the depth of the illumination plane (Supplementary Fig. 3a, Supplementary

10 Movie 1). This increases the full-width at half-maximum intensity and reduces image quality

11 in the brain, compared to images acquired from the relatively thinner neck (Supplementary

12 Fig. 3b). To achieve single-molecule localization in deep tissues, we integrated the lightsheet

13 with optical tissue clearing technology ${ }^{45,46}$. When the fly brain is cleared with neutral

14 ScaleView-A2 solution $^{18}$, the Bessel lightsheet provides enough energy to excite a dense

15 population of HMSiR fluorophores at high efficiency throughout the entire brain. As

16 expected, blinking signals are much clearer in ScaleView-A2 than in PBS, especially beyond

17 a depth of $30 \mu \mathrm{m}$ (Supplementary Fig. 4). Moreover, the localization uncertainties remain invariant as the imaging depth increases (Fig. 1d). This single-molecule DLM imaging

19 method allows for the three-dimensional reconstruction of olfactory projection neurons,

20 whose axons extend from the antennal lobe at the frontal surface of the brain to the calyx at

21 the posterior surface (Supplementary Fig. 5a). With over 50 million molecules localised at a

22 lateral precision of approximately $30 \mathrm{~nm}$, the quality of these DLM images represents a

23 substantial improvement over conventional lightsheet images acquired using the same optical

24 parameters (Supplementary Movie 2). 
1 Localization images were reconstructed using ThunderSTORM ${ }^{19}$, an ImageJ plugin with a

2 self-built macro for batch processing of massive data. As schematically depicted in

3 Supplementary Fig. 2, data were initially transposed to time lapse series on a local

4 workstation, after which they were transferred to remote Lustre storage and distributed to a

5 three-node Torque cluster (Intel Xeon X5660 with 48 GB memory each, connected to Lustre

6 storage). After a particle list was generated for each layer, the list was deposited into one of

7 the clusters, after which a single list containing all localization events within the imaging

8 volume was generated. The list was then used to render the reconstruction image for

9 presentation and analysis. ThunderSTORM was used to perform drift correction using

10 fiducial marker tracking or the cross-correlation method. For three-dimensional volume

11 rendering, the image stack was resampled to minimize discontinuities in structural integrity.

12 We used the Material Statistics module in Avizo 9.4 (Thermo Fisher Scientific Inc) to

13 quantify the volume of MB sectors, DPM neurons and VMAT protein expression. This

14 module calculates the voxel numbers inside a labeled area, which can be transformed into

15 volumes by multiplying by a known voxel size in each image. We manually segmented the

16 boundaries of MB sectors using the Lasso tool in segmentation mode. We used the Magic

17 Wand tool to select one seed within the DPM neuron and to determine a reasonable threshold

18 for selecting connecting voxels. We directly used the threshold tool for whole-volume

19 VMAT images, setting the lower bound to 78 (8-bit; 0-255).

\section{Analysis of image acquisition speed and resolution}

21 Resolution in localization microscopy is governed by localization precision and the density of

22 localization events. When resolving novel structures, an extremely high localization density

23 is required ${ }^{13}$. Very long image acquisition times are required for this outcome, however,

24 which slows experimental throughput. To determine a realistic acquisition time with a

25 reliable statistical basis, we plotted resolution with respect to time. For localization of VMAT 
1 expression in DPM neurons, localization density (number of localization events/area of the

2 structure) increases exponentially with acquisition time. As the number of sampling frames

3 increases, the growth of localization density slows due to photo-bleaching and depletion of

4 dye molecules (Supplementary Fig. 9a). The theoretical resolution limit is estimated by the

5 localization precision and the Nyquist resolution ${ }^{47,48}$. Resolution power increases rapidly in

6 the first 300 frames (Supplementary Fig. 9b). Afterwards, resolution power slows and

7 finally converges at $86 \mathrm{~nm}$. This represents a lower bound of structural resolution that can be

8 achieved with our current methods.

9 More generally, if an infinite photon budget existed and no photo-bleaching occurred, 10 localization density would increase monotonically with the number of sampling frames. Such 11 modeling indicates that resolution reaches $25 \mathrm{~nm}$ when 10,000 frames per layer are used in reconstruction - which is 20 times the acquisition time used in this study. Our observed dependence of resolution on sampling frames reveals an exponential relation (likely due to photo-bleaching and limited photon budget; Supplementary Fig. S9c), which suggests that the acquisition time required for maximal resolution may be much longer.

16 Fourier ring correlation (FRC) provides a quantitative determination of the structural 17 characteristic in the reconstructed image ${ }^{49}$. The FRC value at the $1 / 7$ cutoff frequency in VMAT protein localization is $283.6 \pm 69.9 \mathrm{~nm}$ (Supplementary Fig. 9d). It should be noted that FRC analysis is based on weighting of the spatial frequency domain and is dominated by the primary feature within the image. As shown in the inset of (Supplementary Fig. 9d), VMAT protein presents an island feature with a dimension from hundreds of nanometers to micrometer, which leads to a higher estimated FRC value.

When considering the statistics of localizing VMAT molecules, the distribution of VMAT is confined to DPM axons. Consequently, the reliability of the results is dominated by localization precision rather than structural determinations. Regardless, we kept the number 
1 of frames of time-lapse data used in VMAT localization to 400 to 500 per layer to ensure all

2 analyses were performed based on similar localization density and theoretical resolution.

3 Acquisition time for one VMAT dataset was approximately 5.6 hours (100 ms per frame, 401

4 frames per imaging volume, 500 volumes recorded). For localization of anti-TH signals (Fig.

5 2), acquisition time was approximately 5.8 hours $(80 \mathrm{~ms}$ per frame, 521 frames per imaging

6 volume, 500 volumes recorded). With four sub-volumes per whole brain, total acquisition

7 time took approximately 23.2 hours to complete.

\section{Drosophila memory assay}

9 Flies were subjected to aversive olfactory conditioning 2 to 5 days after eclosion. Prior to 10 conditioning, flies were accommodated to a behavioral room with a temperature of $20^{\circ} \mathrm{C}$ and 11 relative humidity of $70 \%$ for $30 \mathrm{~min}$. At the start of olfactory conditioning, approximately 80

12 flies were transferred to the training tube, after which two aversive odors (3-octanol (OCT); 13 dilution: $1.5 \times 10^{-3}$; Sigma-Aldrich) and 4-methylcyclohexanol (MCH); dilution: $1.0 \times 10^{-3}$; 14 Fluka) were delivered successively in a current of air $(750 \mathrm{ml} / \mathrm{min})$ for $60 \mathrm{~s}$ at intervals of 45 15 s. The first odor (CS+) was paired with 12 pulses of electric foot shock at $65 \mathrm{~V}$ (serving as 16 the unconditioned stimulus [US]), while the second odor (CS-) was not. This process 17 represented a single session of training. In our experiment, we conducted three sessions of 18 spaced training, with a 10-min interval between each cycle.

19 Gal4 expression was inhibited by $G a l 80^{t s}$ by maintaining flies at $18^{\circ} \mathrm{C}$. One day before 20 training, flies were moved from $18^{\circ} \mathrm{C}$ to $30^{\circ} \mathrm{C}$, thereby deactivating Gal80 $0^{t s}$. Control flies

21 were maintained at a constant temperature of $18^{\circ} \mathrm{C}$. Wild-type and experimental flies carrying

22 the same transgenes were trained using several different protocols: three spaced sessions $(3 \mathrm{x}$ 23 spaced), three massed sessions ( $3 \mathrm{x}$ massed), or 10 spaced sessions (10x spaced).

\section{Statistical analysis}


1 For behavioral experiments, control and treatment groups were tested together (balanced and

2 blinded), with sample sizes listed in figure legends. Because performance indices were

3 normally distributed, the significance of each treatment-versus-control paired comparison

4 was tested using a two-tailed Student's t-test, with P values indicated in figures.

5

6 Reference:

745 Richardson, D. S. \& Lichtman, J. W. Clarifying tissue clearing. Cell 162, 246-257 (2015).

846 Chiang, A. S. et al. Three-dimensional mapping of brain neuropils in the cockroach, Diploptera punctata. J Comp Neurol 440, 1-11 (2001).

1047 Shroff, H., Galbraith, C. G., Galbraith, J. A. \& Betzig, E. Live-cell photoactivated 11 localization microscopy of nanoscale adhesion dynamics. Nat Methods 5, 417-423 (2008)

1248 Shim, S. H. et al. Super-resolution fluorescence imaging of organelles in live cells with 13 photoswitchable membrane probes. Proc Natl Acad Sci U S A 109, 13978-13983 (2012).

1449 Nieuwenhuizen, R. P. et al. Measuring image resolution in optical nanoscopy. Nat Methods 10, 557-562 (2013). 